\title{
List-wide control is not entirely elusive: Evidence from picture-word Stroop
}

\author{
Julie M. Bugg • Swati Chanani
}

Published online: 3 June 2011

(C) Psychonomic Society, Inc. 2011

\begin{abstract}
Stroop interference is attenuated in mostly incongruent lists, as compared with mostly congruent ones. This finding is referred to as the list-wide proportion congruence effect. The traditional interpretation refers to the strategic biasing of attention via list-wide control. In mostly incongruent lists, attention is biased away from the irrelevant words, whereas in mostly congruent lists, words are more fully processed. According to the item-specific account, the listwide proportion congruence effect reflects stimulus-driven mechanisms, and not list-wide control. The unambiguous evidence available to date strongly favors the item-specific account. Using a picture-word Stroop task, we demonstrate a list-wide proportion congruence effect for $50 \%$ congruent items that are embedded in mostly incongruent and mostly congruent lists. This novel finding illustrates that the list-wide proportion congruence effect is not entirely dependent on item-specific contributions and supports the list-wide control account. We discuss factors impacting the emergence of listwide control in Stroop tasks.
\end{abstract}

\section{Keywords Cognitive and attentional control · Stroop}

Hundreds of studies have examined Stroop interference, the increased reaction time (RT) to name the ink color on incongruent (e.g., blue in red) relative to congruent (e.g., blue in blue) trials. Yet the mechanisms used to resolve Stroop interference remain the target of rich theoretical debate (MacLeod, 1991). The present study addresses the debate surrounding the mechanism(s) underlying the listwide proportion congruence effect, the robust finding that

J. M. Bugg $(\bowtie) \cdot$ S. Chanani

Department of Psychology, Washington University,

Campus Box 1125, One Brookings Dr,

St. Louis, MO 63130, USA

e-mail: jbugg@artsci.wustl.edu
Stroop interference is attenuated in mostly incongruent (MI) lists, as compared with mostly congruent (MC) lists (e.g., Kane \& Engle, 2003; Lindsay \& Jacoby, 1994; Logan \& Zbrodoff, 1979; Logan, Zbrodoff, \& Williamson, 1984; Lowe \& Mitterer, 1982; West \& Baylis, 1998). The traditional interpretation, the list-wide control account, focuses on an expectancy-driven mechanism that capitalizes on list-level information such as the frequency of occurrence of particular trial types. In MI lists, where incongruent trials are expected, attention is strategically biased away from word reading to minimize interference (e.g., Lindsay \& Jacoby, 1994; Logan, 1980; Logan et al., 1984; Lowe \& Mitterer, 1982). In MC lists, attention is biased toward word reading, because word reading is beneficial on the most frequent (i.e., expected) trial type.

Recently, the list-wide control account has been challenged by the item-specific account of the list-wide proportion congruence effect (Blais \& Bunge, 2010; Blais, Robidoux, Risko, \& Besner, 2007; Bugg, Jacoby, \& Toth, 2008). According to the item-specific account, participants use information signaled by items (e.g., the proportion congruency of the word red) to modulate word reading, and not information signaled by the list. This account is entirely plausible because the typical list-wide proportion congruence design perfectly confounds list-wide and item-specific proportion congruence (i.e., percentage of times the word red is congruent). Moreover, in lists for which $50 \%$ of trials are congruent, an item-specific proportion congruence effect is observed such that Stroop interference is reduced for MI items (e.g., red and blue), as compared with MC items (e.g., green and white) (Jacoby, Lindsay, \& Hessels, 2003). This means that obtainment of proportion congruence effects does not depend on the global biasing of attention on the basis of list-level information (e.g., expectancies). The implication is that list-wide proportion congruence effects may be itemspecific effects in disguise (Bugg et al., 2008). 
An item-specific interpretation of the list-wide proportion congruence effect differs qualitatively from one based on list-wide control; item-specific effects stem from fastacting, stimulus-driven mechanisms, not a putatively strategic or preparatory mechanism that acts globally. Item-specific mechanisms take two forms (Jacoby et al., 2003). One is item-specific control of word reading. Using the example above, word reading would be more strongly dampened upon presentation of red than upon presentation of green (cf. Bugg, Jacoby, \& Chanani, 2010). The second is associative (i.e., contingency) learning whereby stimulus-response contingencies are used to predict the response most frequently associated with a given word, such as saying "blue" when red is presented (Schmidt \& Besner, 2008). Two studies tested the itemspecific account by implementing a design that permitted examination of the list-wide proportion congruence effect independently of item-specific contributions (Blais \& Bunge, 2010, Experiment 1; Bugg et al., 2008, Experiment 1). In both studies, a list-wide proportion congruence effect was not observed when item-specific influences were controlled. Only when item-specific mechanisms could be operating in place of list-wide control was the list-wide proportion congruence effect obtained.

To summarize, extant evidence supports the operation of item-specific mechanisms that affect Stroop interference, independently of any list-wide influence (Jacoby et al., 2003), and the operation of item-specific mechanisms in the context of a list-wide proportion congruence manipulation (Blais \& Bunge, 2010; Bugg et al., 2008). In contrast, there is no published evidence that the list-wide proportion congruence manipulation stimulates use of a list-wide control mechanism that affects the magnitude of Stroop interference, independently of item-specific influences (but see Fernandez-Duque \& Knight, 2008, for evidence of a sustained control mechanism in a task-switching Stroop paradigm). Thus, it might be reasoned that it is time to abandon the concept of list-wide control.

The present study provides evidence in favor of retaining list-wide control. Using a picture-word Stroop task (see Bugg et al., 2010), we implemented a design that is analogous to the Bugg et al. (2008) and Blais and Bunge (2010) colorword Stroop studies. The key element was the inclusion of a set of items that were $50 \%$ congruent in lists that were MC or MI (see Fig. 1). These proportion congruence (PC)-50 items possessed a list-wide bias, but no item-specific bias. A second set of items was $75 \%$ congruent (PC-75) or $25 \%$ congruent (PC-25) and determined the proportion congruency of the list. These items have an item-specific and list-wide bias. For example, a PC-75 item is MC at the item level and resides within a $\mathrm{MC}$ list.

A critical departure from previous studies is that we attempted to bias participants away from using item-specific mechanisms exclusively. Participants may not have implemented list-wide control in previous studies because associative learning provided a reliable and efficient means for responding (Blais \& Bunge, 2010; Bugg et al., 2008). By increasing the size of the stimulus set from two to four for the PC-75/PC-25 items in the present study, we reasoned that associative learning would be less effective. For an incongruent item from the PC-25 set, for instance, three response options exist, instead of one high-contingency response. Even in the presence of this design iteration, the item-specific account predicts that the list-wide proportion congruence effect should be observed exclusively for the PC-75/PC-25 items. According to the list-wide control account, the list-wide proportion congruence effect is not dependent on the contribution of itemspecific influences. Therefore, the effect should be observed for the PC-50 items, in addition to the PC-75/PC-25 items.

\section{Method}

\section{Participants}

Thirty-two undergraduates (12 males), 18-21 years of age, at Washington University in St. Louis participated for credit. All participants had normal or corrected-to-normal vision and provided informed consent.

\section{Design and materials}

A $2 \times 2 \times 2$ within-subjects design was implemented with trial type (congruent vs. incongruent), proportion congruence (MC vs. MI), and item type (PC-75/PC-25 vs. PC-50) as factors. ${ }^{1}$ Fourteen black-and-white line drawings (three birds, cats, dogs, and fish and one pig and seal) were downloaded from http://thecoloringspot.com, and words were superimposed using Powerpoint (for sample stimuli, see Fig. 1). Stimuli were divided into two sets of items, the PC-75/PC-25 items (bird, cat, dog, fish) and the PC-50 items (pig and seal), and these sets were not permitted to cross (e.g., the word pig was never superimposed on a bird) (see also Blais \& Bunge, 2010; Bugg et al., 2008). As is shown in Fig. 1, the PC-75/PC-25 and PC-50 items were intermixed to yield MC (i.e., $71 \%$ congruent) and MI (i.e., 29\% congruent) lists.

\footnotetext{
${ }^{1}$ An additional factor (load) was manipulated. Participants performed MC and MI blocks of Stroop with and without load (secondary tapping), with order counterbalanced. Because the primary aim of the present study was to examine performance under full attention and to facilitate comparison with previous studies, we report only data from no-load blocks. The order in which load and no-load blocks were administered did not significantly interact with the factors of interest (e.g., trial type, proportion congruence, or trial type $\times$ proportion congruence interaction) in the analyses reported herein $(p \mathrm{~s}>.11)$.
} 
Fig. 1 List composition and sample stimuli
List-Wide Proportion Congruence

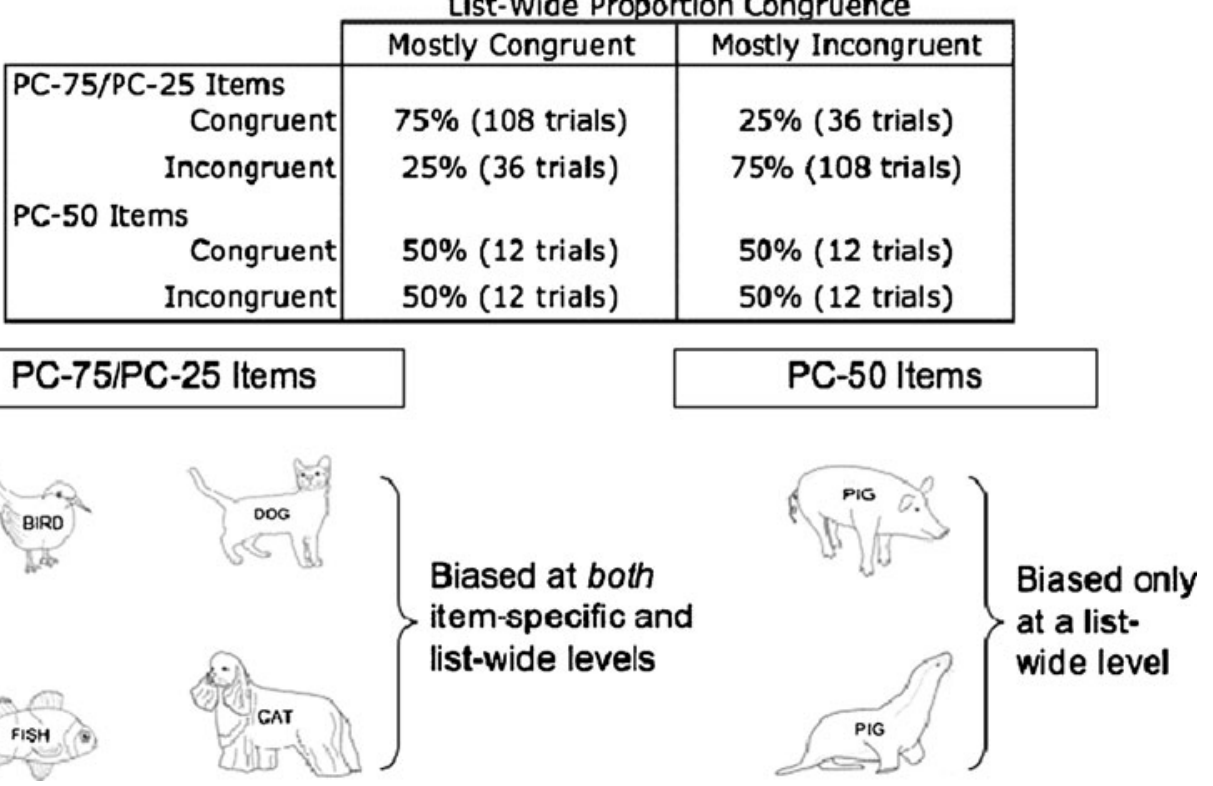

For each item in the PC-75/PC-25 set, there were three possible incongruent pairings (e.g., the word bird superimposed on a picture of a cat, a dog, or a fish). Three different exemplars of the items in the PC-75/PC-25 set were used as pictures. As such, each word in the PC-75/ PC-25 set (e.g., bird, dog) was paired with a total of 12 different pictures (including congruent trials). For each item in the PC-50 set, there was one possible incongruent pairing (e.g., the word pig superimposed on a seal picture). One exemplar of each item in the PC-50 set was used as a picture such that the words pig and seal were paired with a total of two different pictures (including congruent trials). For each item type, all congruent picture-word combinations appeared equally often within each list of trials. The same was true for all incongruent picture-word combinations.

\section{Procedure}

Participants were tested individually and were instructed to name aloud the animal in the picture as quickly and accurately as possible. For each trial, the stimulus was presented via E-Prime in the center of the screen. The pictures and words subtended approximately $20^{\circ}$ and $5^{\circ}$ of visual angle, respectively. The stimulus remained on screen until a response was detected by voice-key. The experimenter entered the participant's response via keyboard, and the next stimulus appeared $500 \mathrm{~ms}$ later. Scratch trials were trials on which the voice-key was tripped by extraneous noise or imperceptible speech. RT(in milliseconds) and error rate were recorded.

Participants completed 24 practice trials prior to the MC and MI blocks of the Stroop task. Block order was counterbalanced across participants. Within a block,
144 PC-75 or PC-25 and 24 PC-50 items were randomly presented. The procedure lasted approximately $45 \mathrm{~min}$. Participants were thanked and debriefed.

\section{Results}

For each condition, correct RTs $>200 \mathrm{~ms}$ were submitted to a recursive outlier trimming procedure (Van Selst \& Jolicœur, 1994), which eliminated, on average, $2.1 \%$ of the trials from each condition. Mean RTs were computed from those that remained. The alpha level was .05 , and partial eta- squared $\left(\eta_{\mathrm{p}}{ }^{2}\right)$ is reported as the measure of effect size. Because the primary goal was to examine whether a list-wide proportion congruence effect would be obtained for the PC-50 items independently of the PC-75/PC-25 items, we conducted a $2 \times$ 2 within-subjects analysis of variance (ANOVA) for each item type, with factors of proportion congruence and trial type (see the PC-75/PC-25 Items and PC-50 Items subsections below). This ANOVA was repeated for error rate. A third factor, order of MC and MI blocks, was included in initial mixed-subjects ANOVAs, but because there were no interactions with this factor ( $p s>.12$ ), we collapsed across it in subsequent analyses.

\section{PC-75/PC-25items}

Reaction time Mean RTs are shown in Table 1. Stroop interference was observed as indicated by the slowing on incongruent trials $(M=715, S E=14)$, as compared with congruent trials $(M=609, S E=13), F(1,31)=363.07$, $M S E=987, p<.001, \eta_{p}^{2}=.921$. The main effect of trial type was qualified by a significant proportion congruence $\times$ trial type interaction, $F(1,31)=58.15, M S E=517, p<.001$, 
Table 1 Mean reaction times (in milliseconds) and error rates (with standard errors in parentheses) as a function of proportion congruence and trial type for the PC-75/PC-25 and PC-50 items

\begin{tabular}{llllll}
\hline & \multicolumn{2}{l}{ Mostly Congruent } & & \multicolumn{2}{l}{ Mostly Incongruent } \\
\cline { 2 - 3 } \cline { 5 - 6 } & Congruent & Incongruent & & Congruent & Incongruent \\
\hline Reaction Time & $594(13)$ & $731(16)$ & & $624(13)$ & $699(13)$ \\
PC-75/PC-25 items & $643(17)$ & $739(18)$ & & $669(16)$ & $726(15)$ \\
PC-50 items & & & & $.017(.004)$ & $.039(.004)$ \\
Error Rate & $.014(.003)$ & $.085(.012)$ & $.019(.006)$ & $.044(.011)$ \\
PC-75/PC-25 items & $.011(.007)$ & $.043(.011)$ & & $.019)$ \\
PC-50 items & & & &
\end{tabular}

$\eta_{p}^{2}=.652$. Consistent with the typical list-wide proportion congruence pattern, Stroop interference was significantly attenuated for the PC-25 items in the MI list $(M=75)$, as compared with the PC-75 items in the MC list $(M=137)$ (see Fig. 2).

Error rate Mean error rates are shown in Table 1. There were significant main effects of trial type, $F(1,31)=47.50$, $M S E=.001, p<.001, \eta_{p}^{2}=.605$, and proportion congruence, $F(1,31)=11.78, M S E=.001, p<.01$, $\eta_{p}^{2}=.275$. Participants made more errors on incongruent trials $(M=.062, S E=.007)$ than on congruent trials $(M=.016, S E=.003)$ and in the MC lists $(M=.050$, $S E=.007)$ than in the MI lists $(M=.028, S E=.003)$. These effects were qualified by a significant proportion congruence $\times$ trial type interaction, $F(1,31)=16.41, M S E=.001$, $p<.001, \eta_{p}^{2}=.346$, indicative of the list-wide proportion congruence pattern. Stroop interference was reduced for the PC-25 items in the MI list $(M=.022)$, as compared with the PC-75 items in the MC list $(M=.071)$.

\section{PC-50 items}

Reaction time Mean RTs are shown in Table 1. The main effect of trial type was significant, $F(1,31)=110.53, M S E=$

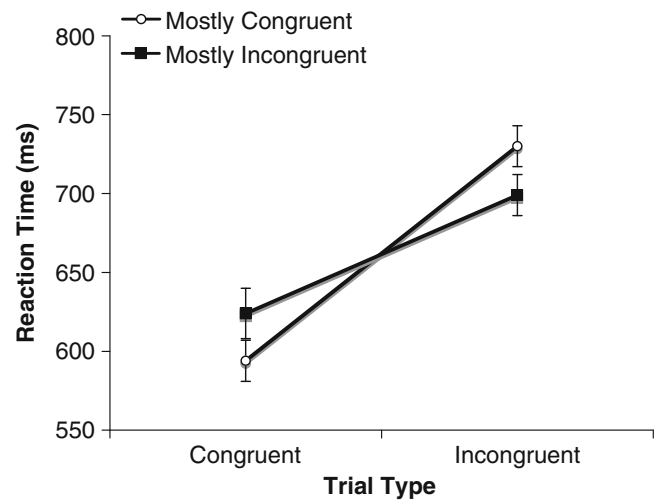

Fig. 2 Mean reaction times as a function of trial type and list-wide proportion congruence for the PC-75 (mostly congruent) and PC-25 (mostly incongruent) items. Bars represent standard errors of the means
$1,702, p<.001, \eta_{p}^{2}=.781$. RTs were longer for incongruent $(M=733, S E=15)$ than for congruent $(M=656, S E=$ $15)$ trials. Most critically, a list-wide proportion congruence effect was observed, as indicated by the significant interaction between proportion congruence and trial type, $F(1,31)=9.07, M S E=1,369, p<.01, \eta_{p}^{2}=.226$. Stroop interference was less pronounced for the PC-50 items that occurred in the MI list $(M=57)$, as compared with the PC50 items that occurred in the MC list $(M=96)$ (see Fig. 3).

Error rate Mean error rates are shown in Table 1. There was a main effect of trial type, $F(1,31)=10.08, M S E=.003$, $p<.01, \eta_{p}^{2}=.245$. Error rate was higher on incongruent $(M=.043, S E=.008)$ than on congruent $(M=.015, S E=$ $.004)$ trials. Unlike for RT, the proportion congruence $\times$ trial type interaction was not significant, $F<1$.

\section{Discussion}

Consistent with previous studies (e.g., Blais \& Bunge, 2010; Bugg et al., 2008; see also Kane \& Engle, 2003; Lindsay \& Jacoby, 1994; Logan \& Zbrodoff, 1979; Logan et al., 1984; Lowe \& Mitterer, 1982; West \& Baylis, 1998), the list-wide proportion congruence pattern was observed for the PC-75/ PC-25 items, items that were biased at the item-specific and

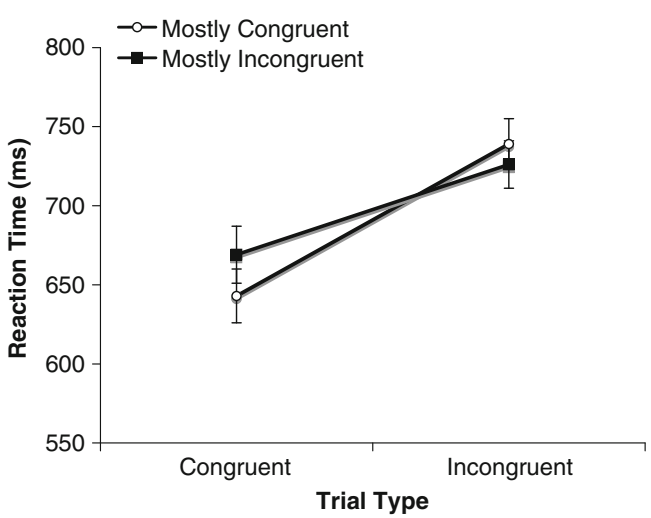

Fig. 3 Mean reaction times as a function of trial type and list-wide proportion congruence for the PC-50 items in the mostly congruent and mostly incongruent lists. Bars represent standard errors of the means 
list-wide levels. Most important, using a set of $50 \%$ congruent items (i.e., PC-50), we demonstrated that a list-wide proportion congruence effect could be observed when item-specific influences are controlled. The PC-50 items, which share neither words nor pictures with the PC-75/PC-25 items, provide no item-level information for participants to modulate word reading or predict high-contingency responses.

The finding that Stroop interference is reduced for PC-50 items in an MI list, relative to the same PC-50 items in an $\mathrm{MC}$ list, can be explained by the operation of a control mechanism that uses information about the proportion congruency of the list to modulate word reading (cf. Lindsay \& Jacoby, 1994). Alternative accounts are, however, possible, because the PC-75/PC-25 items used to create the list-wide biases differ on dimensions other than proportion congruency. Congruent stimuli are repeated more frequently for the PC-75 than for the PC-25 items, and more frequent instances of categorically related incongruent stimuli (dog/cat) occur for the PC-25 than for the PC-75 items. The list-wide control mechanism that affects performance on the PC-50 trials may not be influenced by expectations pertaining to proportion congruency alone but could be influenced by these types of information. With regard to the target of the list-wide mechanism, participants may modulate the word's influence on picture-naming/target-selection. For example, words may be read/processed equally in the MC and MI lists, but reliance on the response activated by the word may differ between the lists.

Others have also demonstrated proportion congruency effects for 50\% congruent items. Crump and Milliken (2009) found a context-specific proportion congruence effect for $50 \%$ congruent items, and Bugg et al. (2010) found an itemspecific proportion congruence effect for $50 \%$ congruent items. These studies did not, however, manipulate list-wide proportion congruence (i.e., all lists were $50 \%$ congruent). To our knowledge, the present finding represents the first demonstration of a list-wide proportion congruence effect for $50 \%$ congruent items. Theoretically, the implication of this novel finding is that list-wide proportion congruence effects are not entirely dependent on the contribution of item-specific mechanisms (cf. Hutchinson, in press). Moreover, our finding demonstrates that traditional accounts that put forth list-wide control as a mechanism for resolving Stroop interference (e.g., Lindsay \& Jacoby, 1994; Logan, 1980; Logan et al., 1984; Lowe \& Mitterer, 1982) should not be abandoned.

Tzelgov, Henik, and Berger (1992) also showed that the Stroop effect is modulated by expectations for interference (incongruent stimuli). In their study, the proportion of congruent to incongruent color words was always $50 \%$, with the key manipulation varying the percentage of neutral trials, relative to color-word trials. As the percentage of neutral trials decreased, the Stroop effect decreased.
According to Tzelgov et al., "evidence of a reduction in the Stroop interference when interference is expected suggests that either the processing of the word is being controlled or the results of its processing are subject to control" (p. 733). Importantly, their manipulation selectively affected incongruent trials, which, according to Tzelgov et al., 'implies that the effect of percentage is 'reactive', that is, information about lexicality is utilized by the control mechanism" (p. 733). That the manipulation selectively affected inhibition (incongruent trials) is contrary to some models of proportion congruence effects (Logan, 1980). For this reason, Tzelgov et al. concluded that "there is no basis to assume that the same processing mechanism is involved in the modulation of the Stroop effect by the proportion of congruent (versus incongruent) stimuli and the proportion of color words versus neutrals" (p. 732). Future research should aim to pinpoint the similarities and differences between the mechanisms underlying the proportion congruence manipulation and other manipulations that alter expectations for interference.

Researchers must be cautious not to assume that any list-wide proportion congruence manipulation will yield measures that reflect list-wide control, because evidence for list-wide control has been elusive in previous studies (Blais \& Bunge, 2010; Bugg et al., 2008). One difference between the present study and previous studies is that we used picture-word stimuli instead of color-word stimuli. Some accounts suggest that different mechanisms underlie interference in picture- and color-word Stroop (Dell'Acqua, Job, Peressotti, \& Pascali, 2007; but see van Maanen, van Rijn, \& Borst, 2009). Accordingly, one might expect that different mechanisms underlie interference resolution across tasks. Notably, however, in an unpublished study, Bugg (2009) reported list-wide control for PC-50 items when using a similar list-wide proportion congruence design in color-word Stroop. Thus, it is unlikely that task type explains the contrasting findings.

Rather, we believe an important difference was our attempt to bias participants away from exclusive reliance on item-specific mechanisms and, in particular, associative learning. Previous studies used a set size of two for the PC75/PC-25 set, whereas we used four items. Associative learning provides a less reliable means of responding in a four-item set where three equally contingent response options exist on incongruent trials than in a two-item set for which there is a single high-contingency response (see Blais \& Bunge, 2010; Bugg et al., 2008). Due to its proactive nature, list-wide control is presumed to be more metabolically costly and resource demanding than itemspecific mechanisms (Braver, Gray, \& Burgess, 2007). Its use may, therefore, be limited to contexts in which itemspecific mechanisms such as associative learning are less effective. Alternatively, or in addition, the increase in list- 
level stimulus variability (due to use of multiple exemplars for the PC-75/PC-25 items) may have increased perceptions of difficulty and stimulated use of list-wide control.

The present findings have important implications for existing theories and computational models. The itemspecific conflict-monitoring model (Blais et al., 2007; cf. Verguts \& Notebaert, 2008) proposes that response conflict is monitored and attention biased at the item level (e.g., frequent presentations of the word bird superimposed on a cat would boost attention to this picture for the word bird). This model has difficulty accommodating the list-wide proportion congruence effect for the PC-50 items because conflict is equivalent for these items in the MC and MI lists. Accordingly, any attentional biasing should have been similar regardless of list, but our data suggest fuller word processing in the $\mathrm{MC}$ list. Alternatively, one might suppose that performance on the $\mathrm{PC}-50$ items reflects carryover effects of item-specific adjustments that occur for the PC-75/PC-25 items, and not adjustments based on list-wide proportion congruence. Item-specific control adjustments, however, affect performance only on subsequent trials for which the word repeats (Blais et al., 2007). Because the words (and pictures) used for the PC-50 items differ from the words used for the PC-75/PC-25 items, the word never repeats when transitioning from a PC-75/PC-25 item to a PC-50 item. The pathwaylevel conflict-monitoring model, in contrast, readily accommodates the list-wide proportion congruence effect for the PC-50 items (Botvinick, Braver, Barch, Carter, \& Cohen, 2001). According to this model, the degree of response conflict within a list results in a global loosening or tightening of control for all items (including PC-50), not just those for which conflict is infrequent or frequent (PC-75 or PC-25).

\section{Conclusion}

Although the present study is not without limitations (e.g., a more ideal design would have rotated the items across conditions), it represents the first demonstration of a listwide cognitive control mechanism that contributes to the list-wide proportion congruence effect independently of item-specific influences in picture-word Stroop. Of theoretical interest is pinpointing contextual features that trigger list-wide control, relative to item-specific mechanisms. The present findings point to the importance of one such feature, the predictability of responses, in moderating the elusiveness of list-wide control.

Author Note Julie M. Bugg and Swati Chanani, Department of Psychology, Washington University in St. Louis.

Data were collected in partial fulfillment of Swati Chanani's honor's thesis. We are grateful to Larry Jacoby, John Heil, Erica Dayan, and Jacqueline Greb for insightful discussion.

\section{References}

Blais, C., \& Bunge, S. (2010). Behavioral and neural evidence for item-specific performance monitoring. Journal of Cognitive Neuroscience, 22, 2758-2767.

Blais, C., Robidoux, S., Risko, E. F., \& Besner, D. (2007). Itemspecific adaptation and the conflict monitoring hypothesis: A computational model. Psychological Review, 114, 1076-1086.

Botvinick, M. M., Braver, T. S., Barch, D. M., Carter, C. S., \& Cohen, J. D. (2001). Conflict monitoring and cognitive control. Psychological Review, 108, 624-652.

Braver, T. S., Gray, J. R., \& Burgess, G. C. (2007). Explaining the many varieties of working memory variation: Dual mechanisms of cognitive control. In A. R. A. Conway, C. Jarrold, M. J. Kane, A. Miyake, \& J. N. Towse (Eds.), Variation in working memory (pp. 76-106). Oxford: Oxford University Press.

Bugg, J. M. (2009). List-wide proportion congruence effects are not always item- specific effects in disguise. Paper presented at the 50th Annual Meeting of the Psychonomic Society, Boston, MA.

Bugg, J. M., Jacoby, L. L., \&Chanani, S. (2010). Why it is too early to lose control in accounts of item-specific proportion congruency effects. Journal of Experimental Psychology: Human Perception and Performance. Advance online publication. doi:10.1037/ a0019957

Bugg, J. M., Jacoby, L. L., \& Toth, J. (2008). Multiple levels of control in the Stroop task. Memory \& Cognition, 36, 1484-1494.

Crump, M. J. C., \& Milliken, B. (2009). The flexibility of contextspecific control: Evidence for context-driven generalization of item-specific control settings. The Quarterly Journal of Experimental Psychology, 62, 1523-1532.

Dell'Acqua, R., Job, R., Peressotti, F., \& Pascali, A. (2007). The picture-word interference effectis not a Stroop effect. Psychonomic Bulletin \& Review, 14, 717-722.

Fernandez-Duque, D., \& Knight, M. (2008). Cognitive control: Dynamic, sustained, and voluntary influences. Journal of Experimental Psychology. Human Perception and Performance, 34, 340-355.

Hutchinson, K. A. (in press). The interactive effects of list-wide control, item-based control, and working memory capacity on Stroop performance. Journal of Experimental Psychology: Learning, Memory, and Cognition.

Jacoby, L. L., Lindsay, D. S., \& Hessels, S. (2003). Item-specific control of automatic processes: Stroop process dissociations. Psychonomic Bulletin \& Review, 10, 638-644.

Kane, M. J., \& Engle, R. W. (2003). Working-memory capacity and the control of attention: The contributions of goal neglect, response competition, and task set to Stroop interference. Journal of Experimental Psychology. General, 132, 47-70.

Lindsay, D. S., \& Jacoby, L. L. (1994). Stroop process dissociations: The relationship between facilitation and interference. Journal of Experimental Psychology. Human Perception and Performance, 20, 219-234.

Logan, G. D. (1980). Attention and automaticity in Stroop and priming tasks: Theory and data. Cognitive Psychology, 12, 523-553.

Logan, G. D., \& Zbrodoff, N. J. (1979). When it helps to be misled: Facilitative effects of increasing the frequency of conflicting stimuli in a Stroop-like task. Memory \& Cognition, 7, 166-174.

Logan, G. D., Zbrodoff, N. J., \& Williamson, J. (1984). Strategies in the color-word Stroop task. Bulletin of the Psychonomic Society, $22,135-138$.

Lowe, D., \& Mitterer, J. O. (1982). Selective and divided attention in a Stroop task. Canadian Journal of Psychology, 36, 684-700.

MacLeod, C. (1991). Half a century of research on the Stroop effect: An integrative review. Psychological Bulletin, 109, 163-203.

Schmidt, J. R., \& Besner, D. (2008). The Stroop effect: Why proportion congruence has nothing to do with congruency 
and everything to do with contingency. Journal of Experimental Psychology. Learning, Memory, and Cognition, 34, $514-523$.

Tzelgov, J., Henik, A., \& Berger, J. (1992). Controlling Stroop effects by manipulating expectations for color words. Memory \& Cognition, 20, 727-735.

van Maanen, L., van Rijn, H., \& Borst, J. P. (2009). Stroop and picture-word interference are two sides of the same coin. Psychonomic Bulletin \& Review, 16, 987-999.
Van Selst, M., \& Jolicœur, P. (1994). A solution to the effect of sample size on outlier estimation. The Quarterly Journal of Experimental Psychology, 47A, 631-650.

Verguts, T., \& Notebaert, W. (2008). Hebbian learning of cognitive control: Dealing with specific and nonspecific adaptation. Psychological Review, 115, 518-525.

West, R., \& Baylis, G. C. (1998). Effect of increased response dominance and contextual disintegration on the Stroop interference effect in older adults. Psychology and Aging, 13, 206-217. 